\title{
Effect of gas flow rate on plasma temperature and electron density of
}

\section{atmospheric argon plasma jet}

\author{
Intesar H. Hashim ${ }^{1}$, Kadhim A. Aadim², Mohammed Rustom Ali ${ }^{2}$ \\ ${ }^{1}$ Department of Physics, College of Education, Al-Mustansiriyah University, \\ Baghdad, Iraq \\ ${ }^{2}$ Department of Physics, College of Science, University of Bagdad, Baghdad, Iraq \\ E-mail: kadhim_adem@scbaghdad.edu.iq
}

\begin{abstract}
In this study, method for experimentally determining the electron density $\left(\mathrm{n}_{\mathrm{e}}\right)$ and the electron temperature $\left(\mathrm{T}_{\mathrm{e}}\right)$ in the atmospheric Argon plasma jet is used; it is based on optical emission spectroscopy (OES). Boltzmann plot method used to calculate these parameters measured for different values of gas flow rate. The results show that the electron temperature decreasing with the increase of gas flow rate also indicates an increasing in the electron density of plasma jet with increasing of gas flow rate.
\end{abstract}

Key words

Atmospheric cold plasma, plasma jet, Boltzmann plot.

Article info.

Received: May. 2017

Accepted: Jul. 2017

Published: Dec. 2017

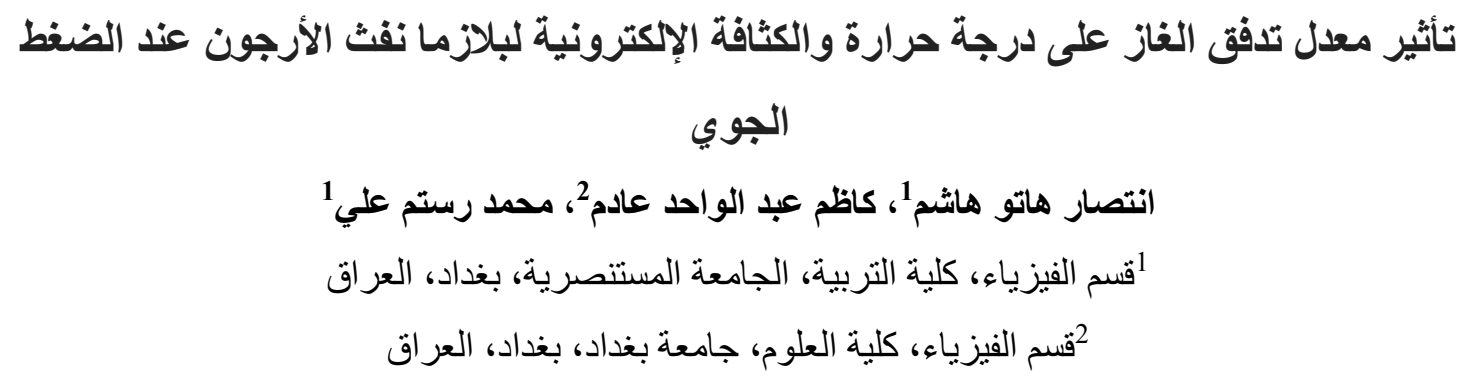

\section{Introduction}

In recent years, Atmospheric Cold Plasma (ACP) achieved increased attention among advanced non-thermal technologies an alternative approach for the elimination of spoilage microorganisms and pathogens from contaminated objects, including fresh and processed food surfaces and medical devices. It has numerous advantages over more conventional methods such as low process operational costs, short treatment time at low temperatures, nontoxic nature [1].

Plasma Jet a promising technique used for generating cold atmospheric pressure glow plasma. Cold atmospheric pressure plasma is extensively used in several plasma processing techniques, such as the surface modification of different 
materials, medical application, air treatment, water treatment, gas treatment [2].

The electrons in the plasma jet are quickly influenced by the external electric-field due to their small masses. In addition, they cannot transfer a large part of their kinetic energy to the heavy particles and hence, they possess higher average kinetic energy when compared with the heavy particles. Electron-impact dissociation, excitation and ionization account for the preliminary reactions in the hierarchy of chemical kinetics. Subsequently, the other chemical processes between heavy particles are initiated. Since electron collisions play an important role in the plasmachemical kinetics, it becomes necessary to determine the electron density in the active plasma volume [3].

The Boltzmann plot is a powerful, simple and widely used method for spectroscopy measurement, especially for measuring the electron temperature of plasma from using the relative intensity of two or more line spectra having a relatively large energy difference. However, in order to practically apply the Boltzmann plot method for the measurement of electron temperature, the excitation level needs to be reached under a local thermal equilibrium (LTE) condition [4].

Boltzmann equation can be explained as:

$\ln \frac{\varepsilon_{j i} \lambda_{i j}}{A_{j i} g_{j}}=-\frac{E_{j}}{K T}+C$

where $\lambda_{i j}$ : wavelength of the emitted light

$\mathrm{A}_{\mathrm{ji}}$ : transition probability

$E_{j}$ : upper energy level

$\mathrm{g}_{\mathrm{j}}$ : statistical weights of the respective state

$\varepsilon_{\mathrm{ji}}$ : emission coefficient of spectral line Eq.(1) can be expressed as: $y=m x+C$

where

slope $=m=-\frac{1}{K T}$

Determination of both the electron density and temperature simultaneously in cold Argon plasma jet by analyzing the Stark broadening of two different emission lines is presented. This method is based on the fact that the Stark broadening of different lines has a different dependence on the electron density and temperature. There for, a comparison of two or more line broadening allows us to diagnose the electron density and temperature simultaneously [5].

Plasma diagnostics is crucial to the understanding of the discharge physics, optimization and the electron density is one of the most fundamental parameters in gas discharges. Typical methods to measure the electron density include the Langmuir probe, Laser heterodyne interferometry, and Laser Thomson scattering, as well as optical emission spectroscopy (OES). The probe method is not suitable for electron density measurements of nonthermal atmospheric pressure plasmas because of the limited size of the discharge and strong collision processes. The complicated and expensive laser system also limits the use of laser diagnostics in real application. In contrast, OES is easy, convenient, and inexpensive and widely used in scientific and applied research [6].

So the electron density can be given by the formula $[7,8]$.

$n_{e}=\exp \left(44.2476+1.20 \ln \Delta \lambda_{1 / 2}-\right.$
$\left.0.6 \ln T_{e}\right)$

where, $T_{\mathrm{e}}$ is the electron temperature in $\mathrm{K}$ and $\mathrm{n}_{\mathrm{e}}$ is the electron number density in $\mathrm{cm}^{-3}$ and $\lambda_{1 / 2}$ is the line width at half maximum intensity of any line, where 
the electron density proportional with $\lambda_{1 / 2}$ (the line width at half maximum intensity of any line).

\section{Experimental}

Atmospheric cold plasma produced using homemade plasma jet system indicates in Fig. 1. Homemade AC power supply used in this work, it produce high voltage reach to maximum value $20 \mathrm{kV}$ with frequency $45 \mathrm{kHz}$. Electron temperature and electron density determined for different values of gas flow rate.

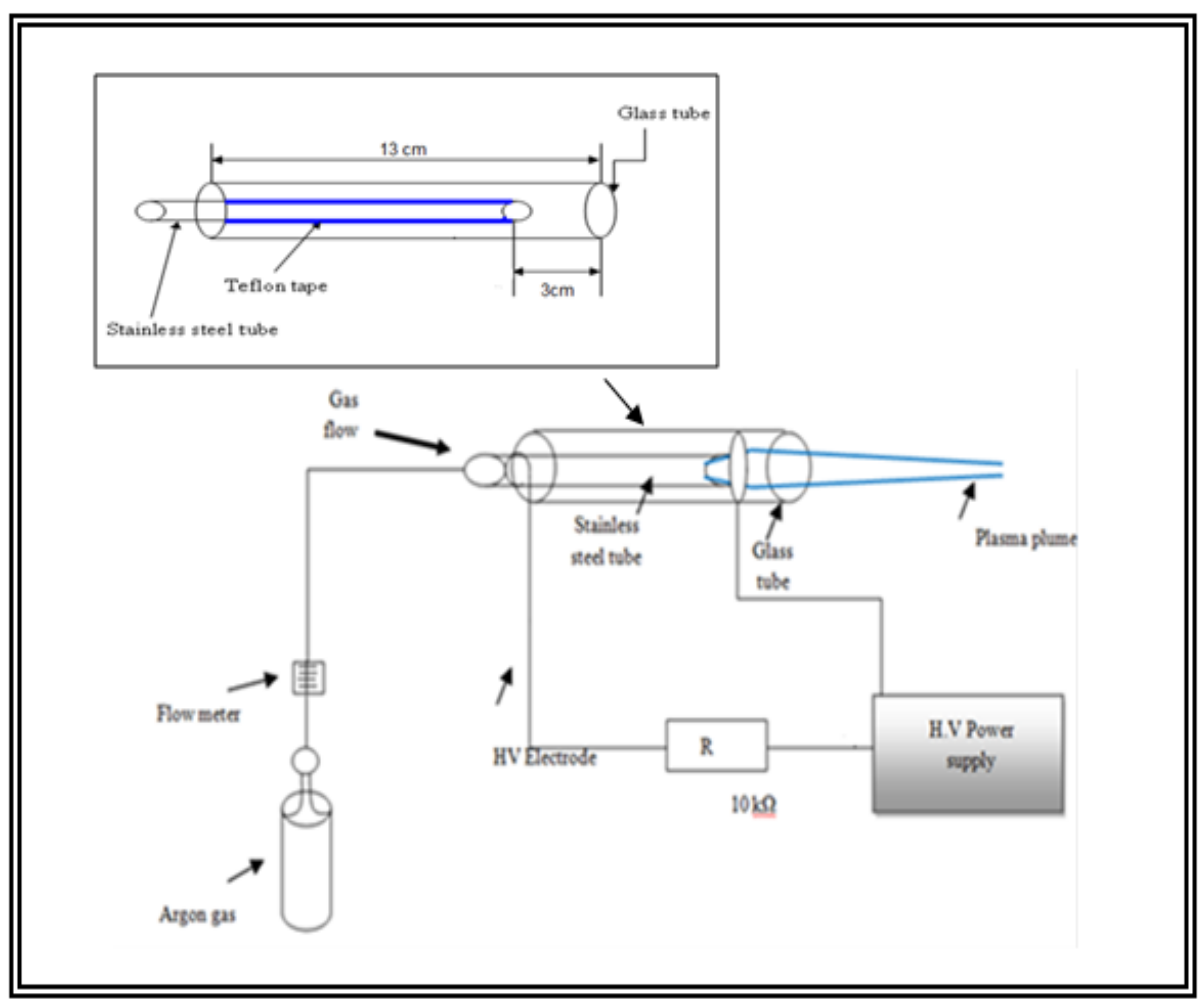

Fig. 1: Plasma jet system.

Optical Emission Spectroscopy (OES) has been used to detect plasma jet composition by observing electronically the excited species and their intensities in the discharges generated by Argon plasma jet. The spectra were recorded in the range (160-1010) nm directly from the plasma jet in axial and radial directions. The electron temperature is determined from the slope of Boltzmann's plot that uses the intensity of several spectral lines versus their corresponding excitation energies. (S3000-UV-NIR) spectrometer used for this purpose is shown in Fig. 2. 


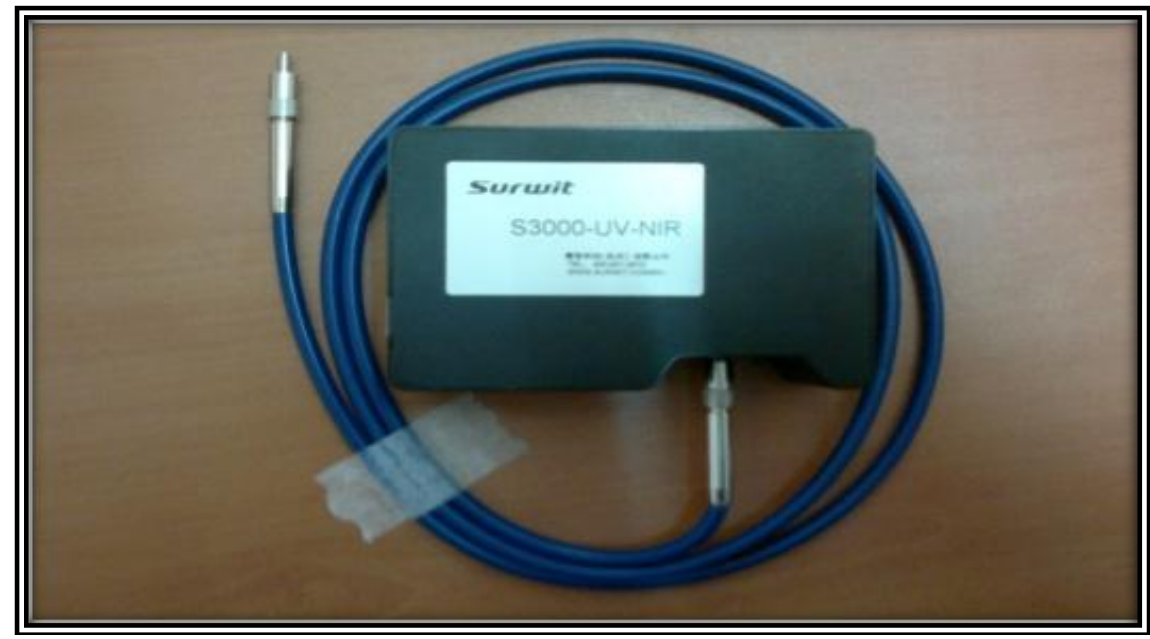

Fig. 2: Optical emission spectroscopy image.

\section{Results and discussion}

At applied voltage $16 \mathrm{kV}$, current $5 \mathrm{~mA}$, frequency $43 \mathrm{kHz}$, and distance $3 \mathrm{~cm}$ for different value of gas flow intensity distribution of plasma rate $(5,10,15,20$ and 25$) 1 / \mathrm{min}$, the spectrum obtained using OES indicates in Fig. 3.

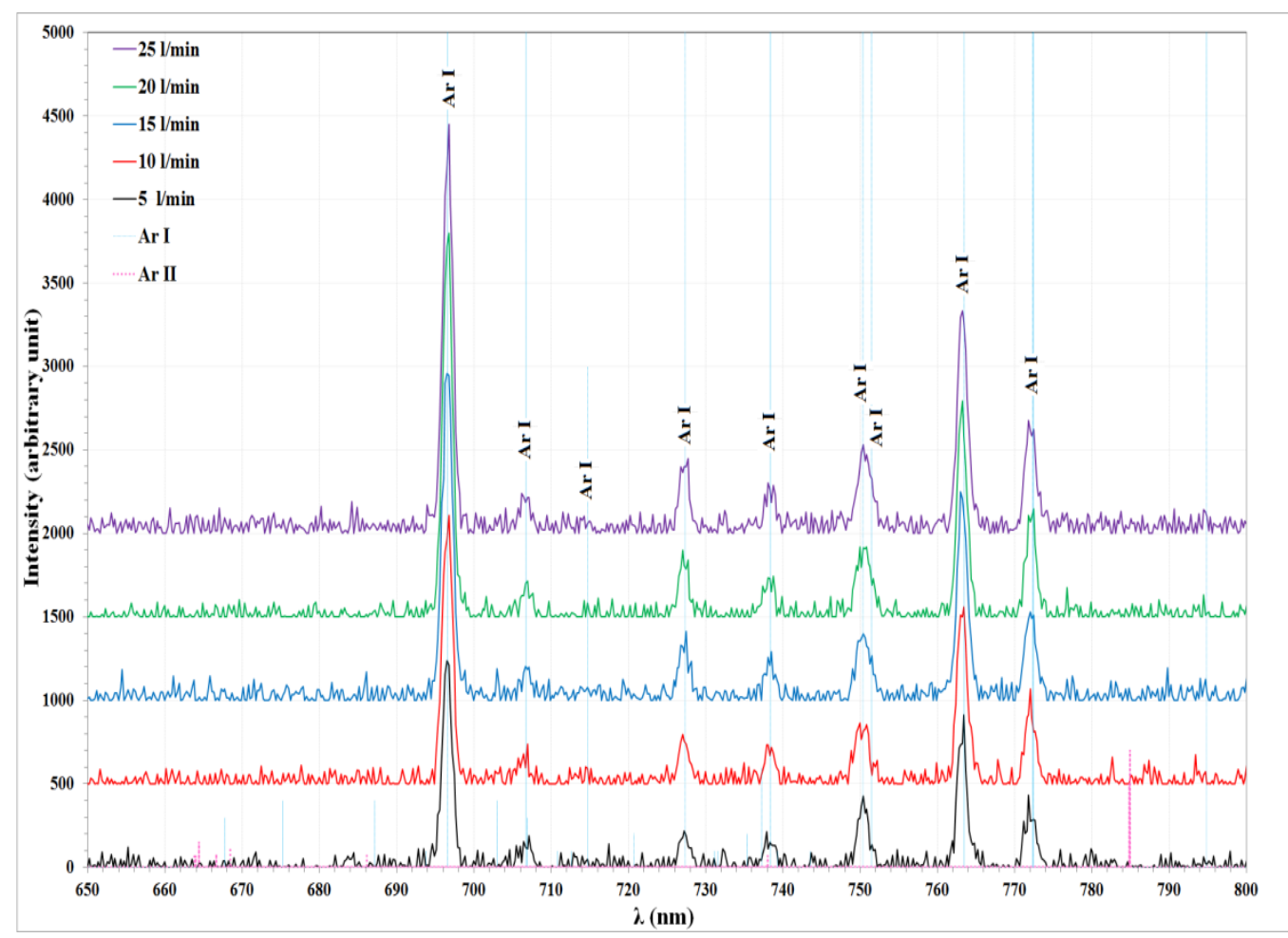

Fig. 3: Variation of intensity with wavelength for different values of gas flow rate.

Many peaks appear in the figure; most these peaks belong to the ArI which agree with NIST data [9]. ArII weak peaks appear in this spectrum. Highest peak appears at $696.5431 \mathrm{~nm}$ for different values of gas flow rate, which indicates the active species generated in the Argon plasma represented by ArI, which agree with [7]. All peaks detected in the visible region (650-800) $\mathrm{nm}$. Also, from the same figure one could be note the 
increasing of peaks high and sharpness with increasing of gas flow rate.

For highest peak as in Fig. 4, it is seen that the increasing of peak intensity with increasing of gas flow rate that is means increasing of ArI species emission in the Argon plasma. While at high value of gas flow rate there is small decreasing in the intensity which may due to the saturation of plasma ionization. Also, the peak half width decrease with increasing of gas flow rate.

For highest peak and by plotting $\ln \frac{\varepsilon_{j i} \lambda_{i j}}{A_{j i} g_{j}} \quad$ verses $\quad \mathrm{E}_{\mathrm{j}}, \quad$ electron temperature could be calculated from the slope of the linear fitting of the result curve indicates in Fig. 5 using Eq. (3). Also, electron density can be also calculated by using the Eq. (4).

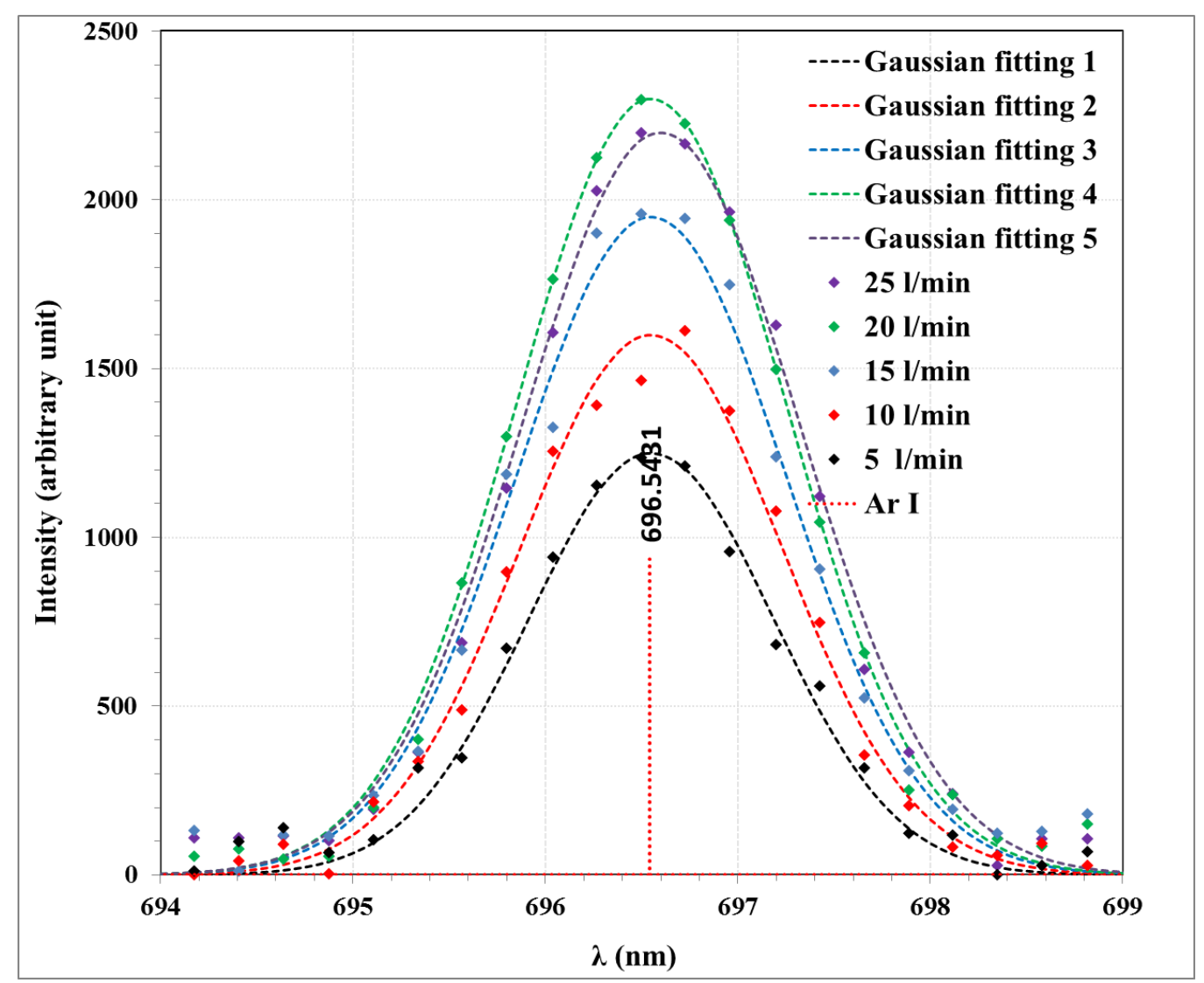

Fig. 4: Variation of intensity with wavelength at highest peak for different values of gas flow rate. 


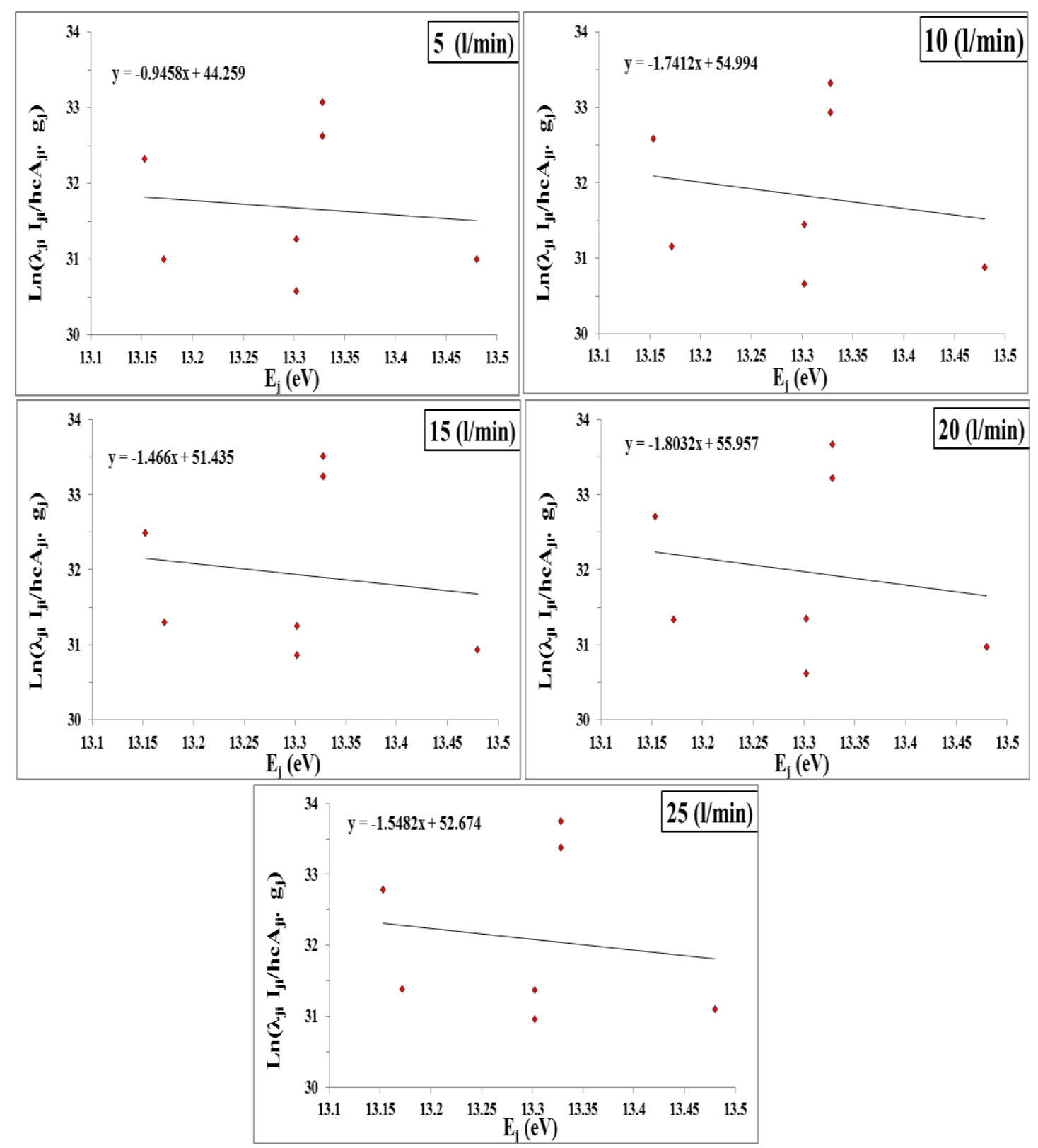

Fig. 5: Boltzmann plot for different values of gas flow rate.

Plotting the term $\ln \frac{\varepsilon_{j i} \lambda_{i j}}{A_{j i} g_{j}}$ in the vertical axis with $E_{j}$ in the horizontal axis, the electron temperature $\mathrm{T}_{\mathrm{e}}$ which is related to the slope of the linear fitting by using Eq. (3), after that the electron density can be also calculated by using the Eq. (4). So we can clarify the results in Fig. 4.
Variation of electron temperature and electron density with gas flow rate indicates in the Fig. 6, from this figure electron temperature decreasing with the increase of gas flow rate, Also same figure indicates an increasing in the electron density of plasma jet with increasing of gas flow rate. These results agree with [7]. 


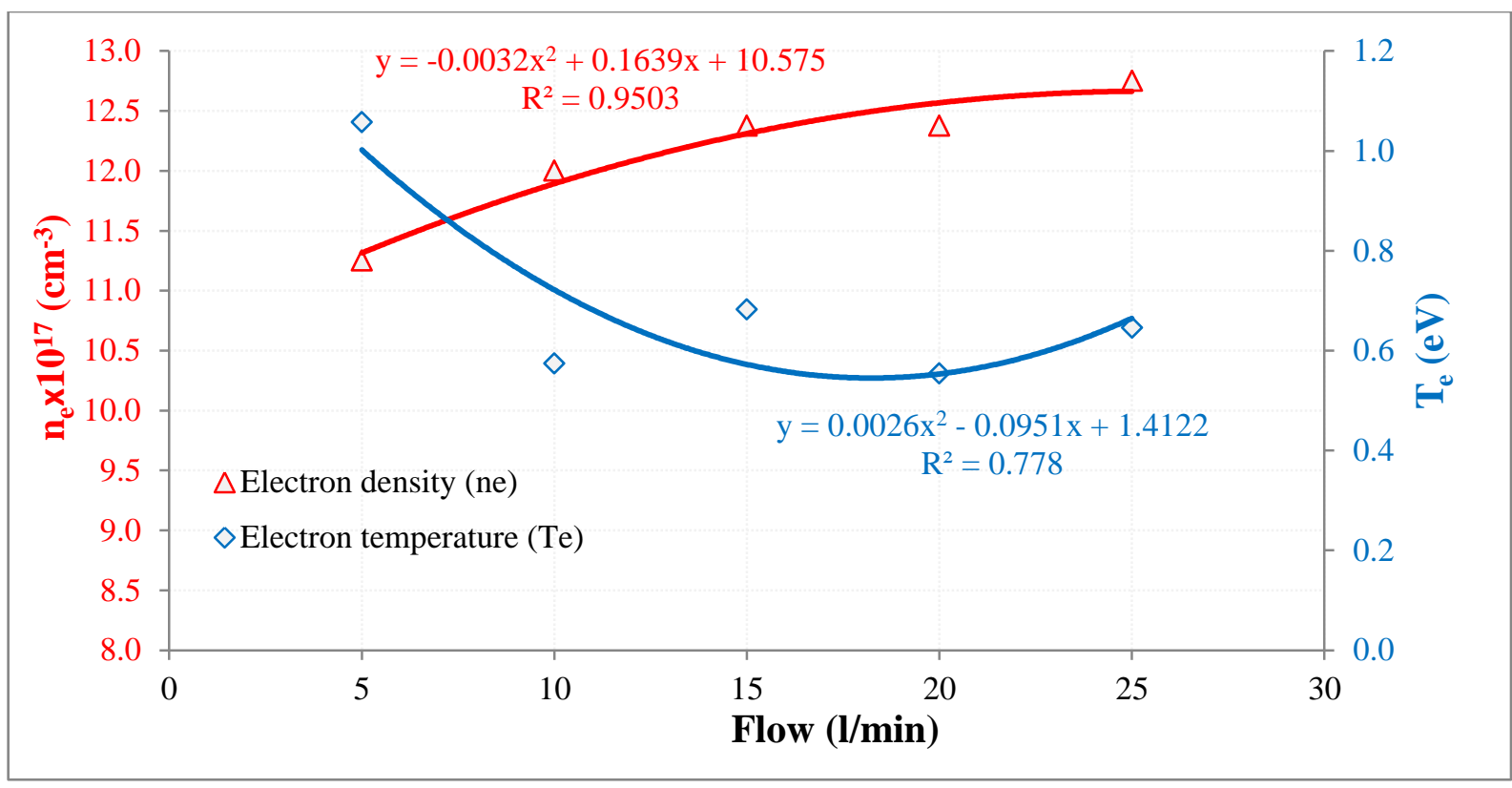

Fig. 6: Variation of electron temperature and electron density with gas flow rate.

That means the increasing of gas flow rate causes an increases in the number of collisions between the electrons and the gas atoms. As a result of these collisions the energy transferred from the electrons to the gas particles increases causing an increase in the gas temperature by decreasing the electron temperature. Also, the mechanism of excitation and ionization of atomic and ionic species in argon plasma is supposed to occur mainly by electron impact. When the gas flow rate increased, the highenergy tail of the electron energy distribution function was contracts to the lower energies. Therefore the direct ionization, which results from the energetic electrons impact with gas atoms, is reduced while the increasing of electron density with flow rate increasing due to the stepwise ionization [7,10].

\section{Conclusions}

Optical Emission spectroscopy measurements indicate that ArI acts as active species generated in the Argon plasma. The results indicates that the electron temperature decreasing with the increase of gas flow rate, that means the increasing of gas flow rate cool down the plasma. Also there is increasing in the electron density of plasma jet with increasing of gas flow rate.

\section{References}

[1] Z. Dana, P. Sonal, B. Paula, K. Kevin, C. Patrick, Journal of Applied Microbiology, 114 (2012) 778-787.

[2] H. H. Murbet, A. Hussein Ali, I. Abdulstar, N. Kaleel A. ALameer, DJBS, 12, 4 (2016) 14-25.

[3] M. K. Boudam, M. Moisan, B. Saoudi, C. Popovici, N. Gherardi, F. Massines, J. Phys. D: Appl. Phys., 39 (2006) 3494-3507.

[4] O. Noriyasu, M. Abdur, U. Hiroshi, T. Shuichi, U. Yoshihiko, Plasma and Fusion Research, 1 (2006) 1-28.

[5] Q. Zhou, Cheng Cheng, Y. Meng, Plasma Science and Technology, 11, 5 (2009) 560-563.

[6] X. Dezhi, Ch. Cheng, Ie. Shen, L. Yan, X. Hongbing, Shu Xingsheng, M. Yuedong, Li Jiangang, P. K. Ch, Physics of Plasma, 21 (2014) 5351053517.

[7] R. H. Hammad, A. A. Qusay, F. R. Aws, International J. of Current 
Engineering and Technology, 5, 6 (2015) 2347-5161.

[8] X. Tu, B. G. Ch'eron, J. H. Yan, K. F. Cen, Plasma Sources Science and Technology, 16 (2007) 803-812.
[9] J. E. Sansonetti, W. C. M. Martin, "Handbook of Basic Atomic Spectroscopic Data", American Institute of Physics (2005).

[10] S. Y. Moon, Physics of Plasmas, 9, 9 (2002) 4045-4051. 